\title{
Double évaluation éthique des projets de recherche menés à l'étranger, en particulier en partenariat Nord-Sud
}

\author{
Groupe de travail \\ «Coordination de l'Evaluation \\ des Essais Cliniques»*
}

\section{* Le groupe de travail «Coordination de l'Evaluation des Essais Cli- niques» ou CEEC réunit, sous le patronage de l'Académie Suisse des Sciences Médicales qui en désigne le président, des représentant(e)s des commissions d'éthique, de Swissmedic, de l'OFSP, des auto- rités cantonales, et de la SSEB. Il s'agit d'un organe de coordination entre l'ensemble des partenaires institutionnels impliqués dans le contrôle et l'évaluation des essais cliniques en Suisse.}

Pour faire face aux besoins croissants de santé publique, la recherche ne peut se limiter aux frontières d'un Etat. La globalisation des échanges touche également de façon naturelle le secteur de la santé. Les activités de recherche tendent ainsi à s'internationaliser. De plus, alors que $90 \%$ de la population mondiale vit dans un pays en développement, seul 10\% de la recherche en santé au niveau international est directement consacré à la lutte contre les maladies et les affections propres à ces pays. Les besoins importants des pays en développement en matière de santé exigent ainsi d'augmenter considérablement les efforts de recherche en partenariat Nord-Sud, à savoir les recherches qui reposent, dans leur conception, sur une communauté d'efforts entre le Nord et le Sud en vue d'atteindre un but mutuellement partagé.

Dans un tel contexte, il est important que chacun des acteurs, en particulier les commissions d'éthique de la recherche (CER), puisse connaître quels sont ses devoirs mais aussi les limites de son activité. La protection des sujets de recherche et la garantie de la qualité des résultats de recherche passent ainsi par une mise en œuvre répétée des principes reconnus d'éthique de la recherche, également lorsqu'ils sont appliqués à des situations nouvelles.

\section{But et champ d'application}

Les présentes propositions sont destinées à aider les CER lorsqu'elles sont confrontées à une demande d'évaluation d'un projet de recherche présentant un élément d'extranéité. Elles définissent quand et à quelles conditions il est nécessaire de procéder à une double évaluation éthique de protocoles de recherche réalisés à l'étranger tout en ayant un lien avec la Suisse. En particulier, il s'agit de recherches conduites en tout ou en partie à l'étranger mais dont le promoteur ou l'investigateur mènent leurs activités depuis la Suisse. Il faut alors déterminer l'étendue de l'évaluation à laquelle doit procéder la CER compétente en Suisse par rapport à celle compétente au lieu de réalisation proprement dit de l'essai (cf. 4).

En principe, ces propositions s'appliquent uniquement aux recherches menées en partenariat Nord-Sud. Il s'agit, selon les directives CIOMS (Council for International Organizations of Medical Sciences), d'études menées dans un pays en développement mais promue, financée et parfois entièrement ou partiellement conduite par une entreprise ou un organisme national ou international d'un pays développé. Ces études peuvent également être financées par un pays en développement mais inclure des chercheurs du Nord.

Ces directives peuvent également valoir pour des études menées en Europe, aux Etats-Unis ou au Japon lorsque la protection des sujets de recherche l'exige: par exemple, lorsque les sujets de recherche sont recrutés activement dans un pays, mais sont déplacés dans un autre pour la réalisation de l'essai proprement dit. Dans ce cas, le contrôle de leur prise en charge et la protection de leurs intérêts doivent faire l'objet d'une attention particulière.

Ce type d'études se différencie des études multicentriques qui sont des recherches réalisées sur la base d'un protocole unique mais dans différents centres de recherche et donc par plusieurs investigateurs. Lorsqu'un essai multicentrique se déroule dans plusieurs pays, il est nécessaire d'obtenir l'avis de la ou des CER compétentes de chaque pays concerné.

\section{Sources}

Le principe d'une double évaluation a été explicitement consacré sur le plan international dans les directives CIOMS dès 1982, puis confirmé lors des révisions de 1993 et 2003. De même, la Déclaration d'Helsinki rappelle que l'investigateur est soumis aux «dispositions éthiques, légales et réglementaires applicables à la recherche sur les sujets humains dans son propre pays ainsi qu'aux règles internationales applicables» alors que le comité d'éthique de la recherche «doit respecter les 
lois et règlements en vigueur dans le pays où s'effectuent les recherches». Si l'investigateur exerce ses activités principales dans un autre pays que celui où se déroule l'étude, il est donc nécessaire de s'assurer que les normes applicables dans les deux pays sont respectées. En Suisse, le code de déontologie de la FMH renvoie explicitement à la Déclaration d'Helsinki en matière de recherche impliquant des êtres humains. Le respect de ce document s'impose donc de manière générale au corps médical comme une obligation professionnelle. De plus, les principales revues scientifiques internationales exigent aujourd'hui une double évaluation éthique pour publier les résultats de recherche en partenariat Nord-Sud. Enfin, au niveau européen, le Protocole additionnel du Conseil de l'Europe sur la recherche biomédicale de 2005 prévoit également que «tout projet de recherche est soumis à un comité d'éthique pour examen indépendant de son acceptabilité sur le plan éthique, dans chacun des Etats où l'une des activités de cette recherche doit avoir lieu».

Aucun de ces textes n'est directement applicable ni contraignant en droit suisse. Néanmoins, la loi sur les produits thérapeutiques (LPTh) exige explicitement le respect des bonnes pratiques des essais cliniques reconnues sur le plan international (art. 53). Le Conseil fédéral a ainsi précisé dans l'ordonnance sur les essais cliniques (OClin) que les essais cliniques de médicaments doivent être conformes aux ICH GCP. Dès lors que les ICH GCP se réfèrent expressément à la Déclaration d'Helsinki, celle-ci s'avère ainsi applicable en matière d'essais cliniques de médicaments. De plus, l'ordonnance sur les exigences relatives aux médicaments (OEMéd) précise que «la documentation sur les essais cliniques doit permettre de démontrer en particulier: a. que les essais sur l'homme ont été effectués selon les règles reconnues des bonnes pratiques des essais cliniques». Même si une double évaluation ne peut en soi être demandée par les autorités suisses, le fait d'avoir obtenu l'avis d'une CER en Suisse constitue donc une mesure utile pour le promoteur afin de s'assurer que le projet en question est effectivement conforme aux ICH GCP et à la Déclaration d'Helsinki. Enfin, le fait pour les CER en Suisse d'assurer le suivi des protocoles de recherche constitue un premier élément dans le monitoring externe et indépendant qui s'avère particulièrement important pour les recherches dans les pays en voie de développement afin de garantir la protection des sujets.

En résumé, il est important que les CER assument leur responsabilité afin de a) répondre aux besoins de santé des pays en développement et b) de préserver la capacité des chercheurs suisses, ainsi que des fonds publics de recherche et de l'industrie de poursuivre leurs activités en partenariat Nord-Sud.

\section{Principe de l'évaluation}

\subsection{Soumission à une commission d'éthique de la recherche}

Tout projet de recherche impliquant des êtres humains doit, avant de débuter, obtenir l'avis favorable de la CER compétente et reconnue, dans chacun des lieux où la recherche est réalisée. Dans le cas d'une recherche en partenariat Nord-Sud, il faut au moins un avis dans un pays du Nord et un avis dans chacun des autres pays où se déroule la recherche. Dans le respect du principe de la souveraineté des Etats, il peut toutefois y avoir plus d'un avis de CER par pays concerné.

Le principe de la double évaluation repose sur la reconnaissance et le respect des règles éthiques et juridiques applicables dans chaque pays concerné. Il découle également de la prise en compte des valeurs propres à chaque communauté et de l'existence de différences culturelles importantes entre les pays dans lesquels se déroulent des recherches en partenariat Nord-Sud. Ces différences doivent donc se refléter aussi dans les CER appelées à procéder à une évaluation. Lorsque ces valeurs sont similaires, par exemple lorsque le promoteur et l'investigateur principal sont domiciliés dans deux pays du Nord, il suffit d'une seule évaluation. Par exemple, lorsque le promoteur a son siège principal en Allemagne et que la recherche se fait en Suisse, il suffit que l'évaluation se fasse par la CER compétente en Suisse.

A défaut d'une législation explicite en la matière, il convient de regarder si, dans la pratique, de telles commissions sont reconnues par des organismes officiels compétents en matière de santé publique et de recherche. En l'absence de commission d'éthique de la recherche dans un pays particulier, il ne peut être entrepris aucune recherche dans le pays en question.

\subsection{Lieu de réalisation d'une recherche}

Une recherche est réputée être réalisée dans chacun des lieux présentant un lien de rattachement avec celle-ci. Il existe un lien de rattachement avec la recherche pour chacune des activités sans lesquelles la recherche ne peut être réalisée. En fait, il y a deux types de rattachements: 
Rattachement principal: le lieu de réalisation proprement dit de la recherche, à savoir:

- le recrutement, la sélection et la prise en charge des sujets dans le cadre de la recherche;

- l'application des procédures testées et le prélèvement de données et d'échantillons biologiques;

- le suivi des sujets après l'étude.

Le rôle essentiel des CER est la protection des sujets de recherche. Toute activité qui a une incidence directe sur cette protection constitue donc un critère de «rattachement principal» pour l'évaluation.

Rattachement secondaire: le lieu où l'investigateur ou le promoteur exercent habituellement leurs activités, par exemple le siège du promoteur ou l'hôpital ou le centre de recherche dans lequel l'investigateur exerce ordinairement sa profession en Suisse.

\subsection{Désignation de la commission}

$\mathrm{Au}$ niveau national, les commissions d'éthique compétentes sont désignées par les législations des Etats dans lesquels le projet doit être soumis pour évaluation. Les règles sur la reconnaissance, la composition et le mode de fonctionnement de ces commissions sont également du ressort de la législation nationale. A défaut, il convient de se référer à la pratique des organismes officiels compétents en matière de santé publique et de recherche (cf. 3.1).

\section{Etendue de l'évaluation}

\subsection{Principe du critère de rattachement}

Pour connaître l'étendue de l'évaluation incombant à chaque CER, il faut prendre en considération le degré de rattachement définissant la compétence de la CER: ceci est déterminant pour chaque CER. Les critères de rattachement ne sont pas tous équivalents (cf. 3.2). L'évaluation éthique a pour but principal la protection des sujets de recherche. Les éléments en lien très étroits avec cet objectif constituent des critères de «rattachement principal». Lorsque le lien avec la protection des sujets est plus ténu, on parle de critères de «rattachement secondaire».

La CER compétente en raison d'un rattachement principal est la mieux placée pour juger des conditions locales susceptibles d'influencer la protection des sujets de recherche. C'est notamment le cas lorsque la compétence de la CER découle du domicile des sujets de recherche, du lieu de l'établissement où se déroule la recherche ou du lieu où le personnel de recherche exerce son activité. Dans une recherche en partenariat
Nord-Sud, ce rattachement concerne principalement les pays du Sud.

La CER compétente en raison d'un rattachement secondaire ne peut pas, ou avec difficulté, avoir connaissance des conditions locales susceptibles d'influencer la protection des sujets de recherche. Elle est ainsi moins à même de pouvoir se prononcer en détail sur le degré effectif de protection des sujets de recherche dans un projet donné. C'est notamment le cas lorsque la compétence de la CER découle uniquement du domicile du promoteur, du coordinateur ou de l'investigateur principal. Dans une recherche en partenariat Nord-Sud, ce rattachement concerne principalement les pays du Nord.

\subsection{Evaluation limitée aux principes}

Lorsqu'un protocole de recherche est soumis à la CER en raison d'un rattachement secondaire, elle limite son évaluation aux principes de base de l'éthique de la recherche tels que consacrés dans la Déclaration d'Helsinki et qui ne sont pas dépendants des conditions locales.

La Déclaration d'Helsinki fixe des standards minimaux au-dessous desquels toute recherche médicale sur des sujets humains est inacceptable. Dans cette mesure, il appartient à toute commission de s'assurer que ces standards sont respectés dans un cas concret. Lorsqu'une commission est saisie en vertu d'un rattachement secondaire, elle doit en particulier vérifier les éléments suivants: le caractère complet du protocole, la validité scientifique de la recherche et sa pertinence médicale, le rapport favorable entre les risques et les bénéfices, l'absence de conflit d'intérêt concernant le promoteur, le coordinateur ou l'investigateur principal, etc. L'examen de principe ne doit pas empêcher la commission de faire une évaluation sur les éléments dépendants des conditions locales, comme le recueil du consentement éclairé des sujets, mais cette évaluation doit se limiter aux principes. Elle doit ainsi examiner si cette procédure est prévue et que l'information transmise est globalement suffisante. Enfin, la CER s'assure que le protocole est effectivement soumis aux CER compétentes dans les pays de réalisation de l'étude. En résumé, la CER en Suisse doit s'assurer que sur le principe une telle étude pourrait se réaliser en Suisse sous réserve que les exigences liées aux conditions locales soient respectées (voir exemple d'avis positif d'une CER en annexe).

\subsection{Evaluation approfondie}

Lorsqu'un protocole de recherche est soumis à la CER en raison d'un rattachement principal, elle devra procéder à une évaluation approfondie. En 
effet, la commission locale est la mieux placée pour évaluer si les conditions et les exigences du pays en question sont respectées. Elle devra s'assurer que, compte tenu des conditions locales, les principes de base de l'éthique de la recherche sont respectés. Il en va ainsi pour le consentement des sujets de recherche, mais également pour d'autres critères comme la qualification du personnel de recherche, la qualité des infrastructures ou les conflits d'intérêts pouvant survenir au niveau local. Lors de cet examen, la commission locale doit en outre avoir accès à l'avis donné par les autres CER compétentes.

\section{Références}

- Déclaration d'Helsinki de l'Association Médicale Mondiale. Principes éthiques applicables aux recherches médicales sur des sujets humains. Paris: WMA; 2000.

- Lignes directrices internationales d'éthique pour la recherche biomédicale impliquant des sujets humains. Genève: CIOMS; 2003.

- Directives des bonnes pratiques cliniques de la Conférence internationale sur l'harmonisation (Directives ICH). 1996.

- Code de déontologie de la FMH. 2006.

- Loi fédérale du 15 décembre 2000 sur les médicaments et les dispositifs médicaux (Loi sur les produits thérapeutiques LPTh).

- Ordonnance du 17 octobre 2001 sur les essais cliniques de produits thérapeutiques (OClin).

- Protocole additionnel à la Convention sur les Droits de l'Homme et la biomédecine, relatif à la recherche biomédicale du Conseil de l'Europe. Strasbourg, 25 janvier 2005.

\section{Annexe}

Exemple d'avis positif concernant un projet de recherche mené à l'étranger

«Sur le principe, le projet de recherche ne présente pas d'éléments contraires aux exigences applicables en matière de recherche impliquant des êtres humains en Suisse concernant en particulier les éléments suivants:
- validité et pertinence scientifique de la conception du projet et des résultats escomptés;

- rapport favorable entre les risques et les bénéfices;

- consentement des sujets de recherche;

- protection de la sphère privée et de la confidentialité;

- existence d'une procédure de compensation des sujets en cas de dommage;

- qualification des chercheurs suisses impliqués dans le projet;

- définition des qualifications requises pour les autres chercheurs.

L'acceptabilité éthique du projet dépend toutefois du respect des conditions locales qui n'ont pas pu être évaluées. Le présent avis ne porte pas en particulier sur les points suivants:

- pertinence du projet du point de vue des besoins en santé publique locaux;

- procédure de recrutement, d'information et de collecte du consentement des sujets de recherche, en particulier les formulaires d'information et de consentement dans le langage local;

- couverture adéquate des dommages subis par les sujets (nature des dommages couverts et montant prévu pour les réparations);

- adéquation des conditions locales du projet (matériel, locaux, personnel, etc.) afin de réaliser le projet tout en respectant la protection des sujets;

- qualification des chercheurs autres que les Suisses.

L'ensemble de ces éléments doivent faire l'objet d'une évaluation approfondie par la ou les CER compétentes désignées $\mathrm{au}(\mathrm{x})$ lieu(x) de réalisation du projet.»

Ces précisions peuvent être directement intégrées dans la fenêtre «conditions» du formulaire d'avis de la CER. 